\title{
Aus den Verhandlungen des FMH-Zentralvorstands
}

St. An seiner Sitzung vom 16. Juni 2004 behandelte der FMH-Zentralvorstand unter anderem folgende Geschäfte.

\section{Qualitätsmanagement}

\section{Europäisches Praxis-Assessment}

Das Projekt «Europäisches Praxis-Assessment» (EPA), welches vom Ressort QM der FMH unterstützt wird, kommt gut voran. Die Pilotstudie zur Validierung der Indikatoren ist durchgeführt, momentan werden die Daten auf europäischer Ebene aggregiert und analysiert. In der nächsten Phase geht es um die Überführung des Projektes in eine nachhaltige Plattform für Qualitätsentwicklung im ambulanten Versorgungssektor. In Deutschland wurde zu diesem Zweck der Verein Praxistest gegründet, der Praxen nach erfolgreicher Durchführung eines EPA-Assessmentprogrammes ein Zertifikat verleiht. In der Schweiz möchten verschiedene Gruppen das EPA-Instrument einsetzen, u.a. die EQUAM. Für diesen Zweck wird momentan eine Struktur geschaffen. Aktuell steht die bereits innerhalb der FMH-Qualitätskommission diskutierte Überführung des Projektes in eine Online-Datenbank für Grundversorgerpraxen zur Diskussion.

Der Zentralvorstand beschliesst eine Anschubfinanzierung von Fr. $40000 .-$, um die Lizenz der Software VISIOTOOL des deutschen Aqua-Institutes zu erwerben.

\section{Prävention}

Nationales Forum Alter und Migration Am 1. Oktober 2004 soll die 2. Nationale Tagung Alter und Migration stattfinden. Die Veranstaltung richtet sich an involvierte Berufsgruppen einerseits, an Migranten andererseits.

Der Zentralvorstand unterstützt diese Tagung und übernimmt eine noch auszuhandelnde Defizitgarantie von maximal Fr. 5000.-.

\section{Aus-, Weiter- und Fortbildung}

\section{Verordnung über Mindestvorschriften für die Anerkennung von Bildungsgängen und Nachdiplomstudien an höheren Fachschulen}

Der Entwurf zu dieser Verordnung, welche einen grossen Teil der paramedizinischen Berufe umfasst, regelt einzig die Bildungsgänge und die Anerkennung der Schulen - das Berufsbild inklusive Kompetenzen und Fähigkeiten, die daraus folgen, werden nicht erwähnt. Dafür sollen offenbar Rahmenlehrpläne - unter Einbezug der sogenannten Organisationen der Arbeitswelt erarbeitet werden. Im Hinblick auf Abgrenzungsprobleme der paramedizinischen Berufe gegenüber der Ärzteschaft soll angeregt werden, auch im Rahmen dieser Verordnung auf diesen kritischen Punkt hinzuweisen.

Der Zentralvorstand begrüsst die Möglichkeit eines speziellen zur Fachhochschulreife führenden Bildungsangebotes. Er hält fest, dass Praktika namentlich in den Gesundheitsberufen unbedingt erforderlich und nach wie vor zeitgemäss sind. Der Zentralvorstand stimmt dem Konzept und der Vorgehensweise der Titelvergabe $\mathrm{zu}$; zur Vermeidung von Missverständnissen sollte allenfalls das Kürzel «dipl.» beigefügt werden können. Er schlägt vor, schon heute folgende weitere Bildungsgänge/Titel vorzusehen: Elektrophysiologisch-technische Assistentin/Assistent sowie Osteopathin/Osteopath. Der Erlass von Rahmenlehrplänen, unter Einbezug der betroffenen Kreise, ist für den ZV unbedingt erforderlich; dies nur schon, weil sich die vorliegende Verordnung mehr oder weniger darauf beschränkt, Stundenzahlen zu definieren. Für jeden einzelnen Bildungsgang soll ein klares Berufsbild innerhalb der Gesundheitsversorgung definiert werden und davon abgeleitet die beruflichen Kompetenzen, verstanden als fachliche, methodische, sozial-kommunikative und persönliche Kompetenzen. 


\section{Organisatorisches}

\section{Übergaberapport des FMH-Präsidenten}

Dr. H. H. Brunner übergibt alle Präsidialgeschäfte dem Zentralvorstand, der für die Weitergabe an den neuen Präsidenten verantwortlich zeichnet. Der Übergaberapport des Präsidenten wird Punkt für Punkt besprochen und genehmigt. Ausserdem genehmigt der ZV den Übergaberapport, der von der Leiterin des Vorstandssekretariats, Denise Rüegg, erstellt worden ist.

\section{Délibérations du Comité central de la FMH}

St. Lors de sa séance du 16 juin 2004, le Comité central a traité, entre autres, des affaires suivantes:

\section{Gestion de la qualité}

\section{Evaluation européenne des cabinets}

\section{médicaux}

Le projet «Evaluation européenne des cabinets médicaux», soutenu par la FMH et plus précisément par son département Gestion de la qualité, avance bien. L'étude pilote pour la validation des indicateurs est terminée; les données sont maintenant agrégées et analysées sur le plan européen. L'étape suivante consistera à transférer le projet dans une plate-forme durable pour le développement de la qualité dans le secteur ambulatoire des soins. A cet effet, l'Allemagne a créé l'association «Praxistest», laquelle délivre un certificat aux cabinets médicaux qui ont participé avec succès au programme d'évaluation européenne. En Suisse, différents groupements souhaitent appliquer cet instrument d'évaluation, notamment la fondation EQUAM. Une structure spécifique est en train d'être créée dans ce but. A la FMH, la Commission en charge de la qualité examine actuellement le transfert du projet dans une banque de données en ligne recensant les cabinets des médecins de premier recours.
Le Comité central décide d'un montant définitif de financement de Fr. 40 000.- pour acquérir la licence du logiciel VISIOTOOL mis au point par l'institut allemand Aqua.

\section{Prévention}

Forum «âge et migration»

Le $1^{\text {er }}$ octobre 2004 se tiendra le $2^{\text {ème }}$ forum «âge et migration». Cette manifestation s'adresse aux groupes de travail concernés, d'une part, et aux migrants, d'autre part.

Le Comité central soutient ce forum et garantit une prise en charge du déficit, qui reste encore à définir, à hauteur maximale de Fr. 5000.-.

\section{Formation prégraduée, postgraduée et continue}

Ordonnance concernant les conditions minimales de reconnaissance des filières de formation et des études post-diplôme dans les écoles supérieures

Ce projet d'ordonnance qui englobe une grande partie des professions paramédicales, règle uniquement les filières de formation et la reconnaissance des écoles. Le profil professionnel, ainsi que les compétences et aptitudes qui en ré- 
sultent, ne sont pas concernés. Celles-ci seront apparemment définies au sein de plans d'études cadres avec l'aide des organisations du monde du travail. En ce qui concerne les problèmes de délimitation des professions paramédicales par rapport aux médecins, il convient d'obtenir que ce point critique soit également pris en compte dans cette ordonnance.

Le Comité central salue la possibilité de suivre une formation spéciale donnant accès à un diplôme de haute école. Il souligne que les stages, notamment dans les professions de la santé, sont absolument nécessaires et qu'ils restent adaptés à notre temps. Le Comité central approuve le concept et les modalités réglant l'octroi des titres; pour éviter tout malentendu, il faudrait pouvoir ajouter éventuellement l'abréviation «dipl.». Il propose de prévoir, aujourd'hui déjà, les nouvelles formations/titres suivants: assistant/e technique en électrophysiologie et ostéopathe. Le Comité estime en outre qu'il est indispensable de mettre au point et de publier des plans d'études cadres, en collaboration avec les milieux concernés, car le présent projet d'ordonnance se limite plus au moins à fixer le nombre d'heures d'enseignements. Pour chaque formation, il faut définir le profil professionnel dans le cadre de l'assistance médicale puis en faire découler les compétences professionnelles, comprises comme compétences spécialisées, méthodiques, socio-communicatives et personnelles.

\section{Organisation}

Rapport sur la remise des dossiers par le président de la FMH

Le Dr H.H. Brunner remet tous les dossiers au Comité central, lui-même responsable de leur transmission au nouveau président. Le rapport du président sortant est discuté et approuvé point par point. Le Comité central approuve également le rapport établi par Denise Rüegg, responsable du secrétariat de direction. 